\title{
Lobulated esophageal schwannoma resected with concurrent approach from the thorax and cervix
}

\author{
Yoshinori Iwata ${ }^{1 *}$, Chihiro Tanaka ${ }^{1}$, Shuji Komori ${ }^{1}$, Narutoshi Nagao ${ }^{1}$, Masahiko Kawai ${ }^{1}$, Kazuhiro Yoshida ${ }^{2}$ \\ and Katsuyuki Kunieda ${ }^{1}$
}

\begin{abstract}
Background: Esophageal schwannomas are rare esophageal submucosal tumors. We herein report a case of a lobulated esophageal schwannoma resected with concurrent approach from the thorax and cervix.

Case presentation: A 74-year-old woman visited our hospital with complaint of loss of consciousness, and a lobulated mediastinal tumor was discovered by chance in computed tomography. Upper gastrointestinal endoscopy showed a smooth elevated lesion at a position of 23-28 cm from the incisor teeth. A hypermetabolic appearance was noted on positron emission tomography. Based on these data, a gastrointestinal stromal tumor was suspected. The tumor was enucleated at the thoracic cavity while being pushed from the cervical incision. Pathological examination showed an esophageal schwannoma.
\end{abstract}

Conclusions: We experienced a case of lobulated esophageal schwannoma with fluorodeoxyglucose accumulation. We resected the tumor with concurrent approach from the thorax and cervix.

Keywords: Esophageal schwannoma, Esophageal submucosal tumor, Mediastinal tumor, Positron emission tomography, Thoracotomy

\section{Background}

Most esophageal tumors are cancer including squamous cell carcinoma and adenocarcinoma, and benign tumors are less than $1 \%$ of all esophageal tumors [1]. Leiomyoma is more than half of all benign esophageal tumors, and schwannoma is rare $[1,2]$. The most common treatment for esophageal submucosal tumor is enucleation by surgery or endoscopy [3]. Recently, the cases of esophageal submucosal tumor resected by thoracoscopy have increased [4]. But the most important point in surgery is safety and curability, so we have to select which approach is better thoracoscopy surgery or thoracotomy by size of the tumor. We herein experienced a case of esophageal schwannoma resected with concurrent approach from the thorax and cervix.

\footnotetext{
* Correspondence: yoshinori_vb2@yahoo.co.jp

'Department of Surgery, Gifu Prefectural General Medical Center, 4-6-1

Noisshiki, Gifu, Japan

Full list of author information is available at the end of the article
}

\section{Case presentation}

A 74-year-old woman visited our hospital with complaint of loss of consciousness, and a mediastinal tumor was discovered by chance in computed tomography $(\mathrm{CT})$. A chest $\mathrm{CT}$ showed a lobulated tumor with a maximum diameter of $8 \mathrm{~cm}$, which was located at upper mediastinum extending to cervix and was compressing the esophagus (Fig. 1a, b). Upper gastrointestinal endoscopy showed a smooth elevated lesion at a position of $23-28 \mathrm{~cm}$ from the incisor teeth (Fig. 2). Magnetic resonance imaging (MRI) showed uniformity and clear boundary tumor (Fig. 3a, b). A hypermetabolic appearance (maximum standardized uptake value, 15.0) was noted on positron emission tomography (PET) (Fig. 4). Based on these data, a gastrointestinal stromal tumor was suspected. The patient was placed in the left lateral position and underwent anterolateral thoracotomy via the sixth right intercostal space. At the same time, the skin incision was added in the right side of the cervix. The 


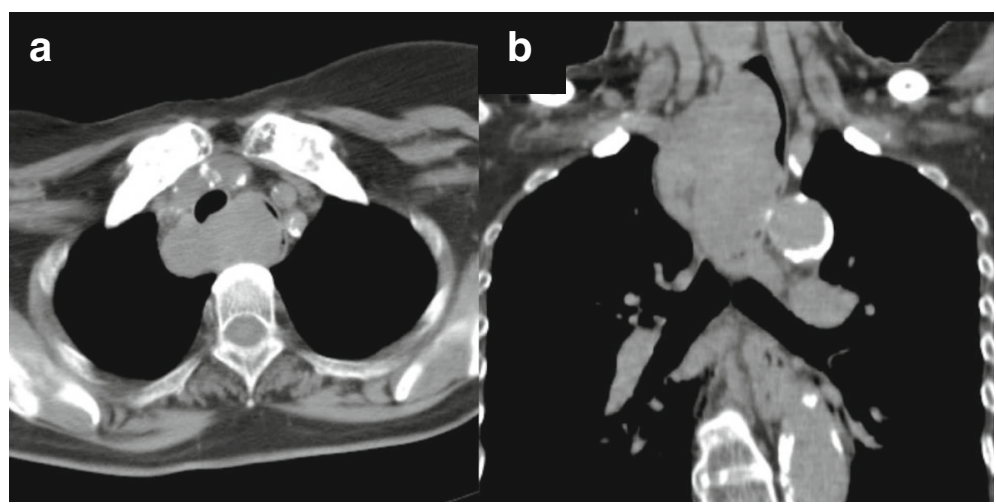

Fig. 1 Computed tomography $(C T)$. a, b A chest CT showed a lobulated mediastinum tumor extending to the cervix

tumor was enucleated at the thoracic cavity while being pushed from the cervical incision (Fig. 5). The muscular layer of esophagus was repaired with sutures. The operation time was $245 \mathrm{~min}$, and the blood loss was $551 \mathrm{~g}$. The specimen showed a welldemarcated, elastic hard, and lobulated appearance and was measured $80 \times 42 \mathrm{~mm}$ (Fig. 6a). The cut surface was almost uniformly pale yellow (Fig. 6b). Hematoxylin and eosin staining revealed spindleshaped cells configuring plexiform proliferation (Fig. 7a). Immunohistochemical examination revealed S-100 protein positivity (Fig. $7 \mathrm{~b}$ ) and c-kit, $\alpha$-smooth muscle actin, desmin, and CD34 negativity, establishing the diagnosis of esophageal schwannoma. The MIB-1 labeling index was $<10 \%$. Her postoperative course was uneventful, and no recurrences have seen for 5 years after surgery.

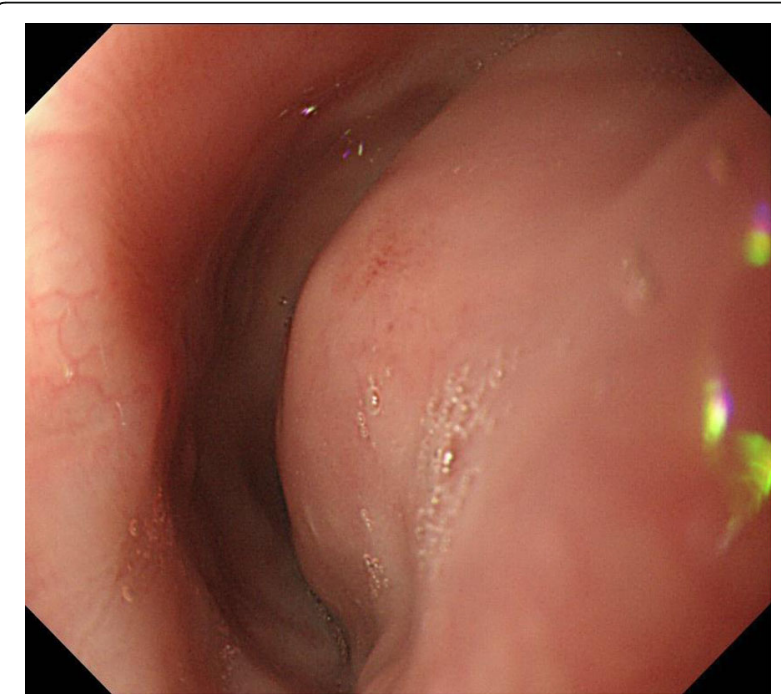

Fig. 2 Upper gastrointestinal endoscopy. Upper gastrointestinal endoscopy showed a smooth elevated lesion

\section{Discussion}

Most esophageal tumors are cancer including squamous cell carcinoma and adenocarcinoma, and benign tumors are less than $1 \%$ of all esophageal tumors [1]. Leiomyoma is more than half of all benign esophageal tumors, and schwannoma is rare [1, 2]. Esophageal schwannoma more frequently develops in women than in men, and these tumors are often located in the upper and mid-esophagus in the mediastinum [5]. Some patients with esophageal schwannoma complain a variety of symptoms including dysphagia, dyspnea, chest pain, and coughing [6,7]; others complain no symptom until the tumors grow larger.

Fluorodeoxyglucose (FDG)-PET as well as CT and MRI are reportedly useful for the confirmation of mediastinal tumors. FDG-PET is usually used to predict the malignancy potential of the tumor or to confirm the recurrence site of the cancer. Many cases of esophageal gastrointestinal stromal tumor with FDG accumulation are reported [8, 9]. In our case, we diagnosed esophageal gastrointestinal stromal tumor for the reason of esophageal submucosal tumor with FDG accumulation. Meanwhile, some esophageal schwannomas with FDG accumulation are reported [10-12]. Schwannomas originate from nerve cells that express glucose transporter type 3, and FDG uptake is considered to be increased for this reason [13]. Preoperative diagnosis of esophageal submucosal tumor is difficult with only imaging findings. On the other hand, EUS-FNA might be able to provide a diagnosis. In general, histological features of schwannoma include spindle-shaped tumor cells arranged in a palisading pattern or with loose cellularity in a reticular array. Immunohistochemical stainings are also useful as schwannoma shows S-100 protein positivity [14]. Although we thought we should undergo EUS-FNA because the huge mediastinal tumor with high FDG uptake had malignancy potential, the patient desired preserving esophagus regardless of the result of the EUS-FNA. 


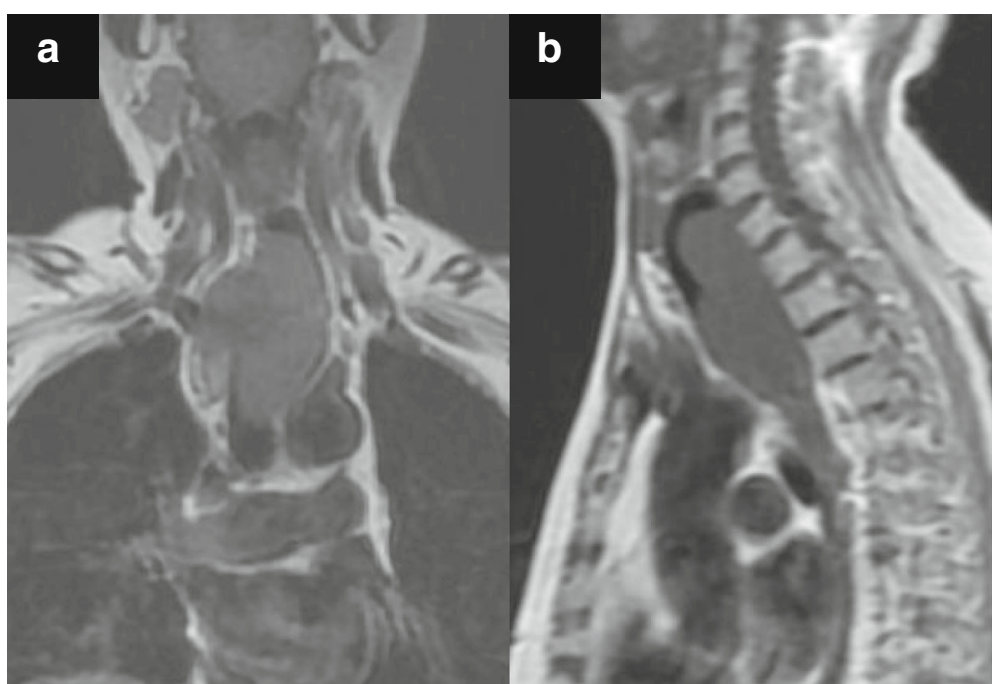

Fig. 3 Magnetic resonance imaging. a, b MRI showed uniformity and clear boundary tumor

Chemotherapy and radiation therapy are ineffective, and the most common treatment is enucleation by surgery or endoscopy for esophageal schwannoma [3]. In general, thoracoscopic resection is advantageous because it is less invasive, therefore shortening

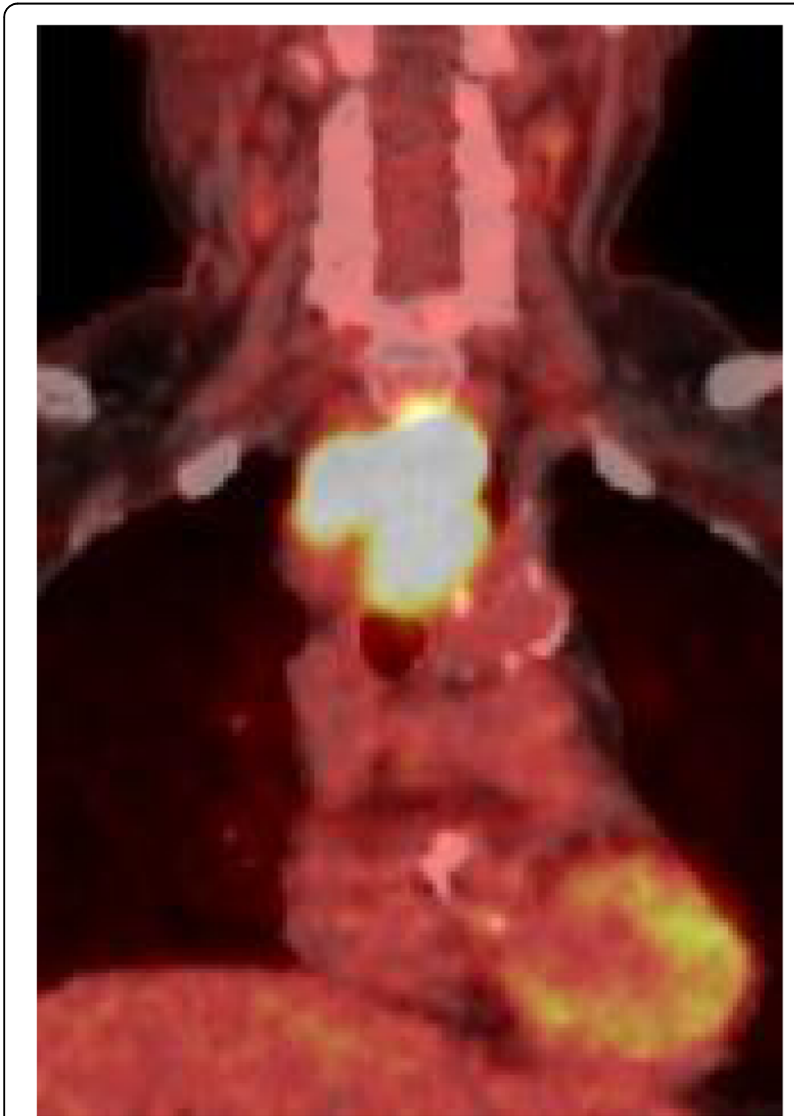

Fig. 4 Positron emission tomography. Positron emission tomography showed hypermetabolic appearance the hospital stay and reducing pain at the surgical wound site [15]. A retrospective study showed that minimally invasive resection of benign esophageal tumors is technically safe and associated with a shorter length of stay compared with open approaches [4]. Although no specific cutoff for size could be identified, most tumors greater than $7 \mathrm{~cm}$ were removed by thoracotomy [4]. In our case, we selected thoracotomy because of the size and shape of the tumor. In addition, as the tumor extended to the cervix, we thought it was difficult to resect the lobulated tumor only from the thorax. We try a concurrent approach from the thorax and cervix to secure the safety and curability. We supposed pushing the tumor from the cervix helped deciding the boundary of the tumor. Actually, the tumor was enucleated at the thoracic cavity while being pushed from the cervical incision with the patient placed in the left lateral position (Fig. 5). We needed an interrupting suture for the defect of muscular layer of esophagus and placed a chest drain near the suture. We removed the chest drain on postoperative day (POD) 6. We performed upper gastrointestinal series (Fig. 8) on POD 9 and found no leakage, slight stenosis in the upper thoracic esophagus, and dilatation in cervical esophagus. We started feeding on POD 10 and found no difficulty in swallowing. We thought endoscopy in the early postoperative period is not needed if the patient has no difficulty in swallowing. The patient was discharged without complications on POD 17. About the follow-up schedule for benign esophageal schwannoma, there has been no consensus yet. We have to follow the patient carefully because of the possibility of recurrence. We followed the patient with CT every 6 months and 


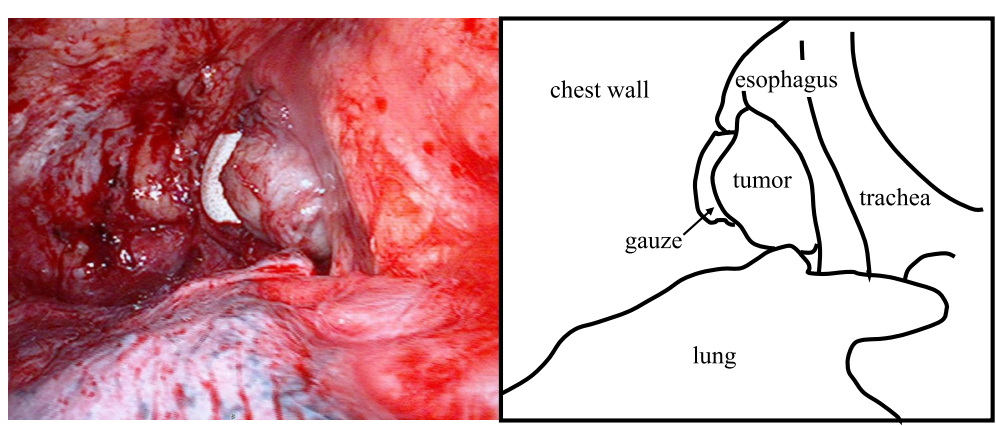

Fig. 5 Intraoperative picture. The tumor was pushed by the gauze inserted from cervical incision

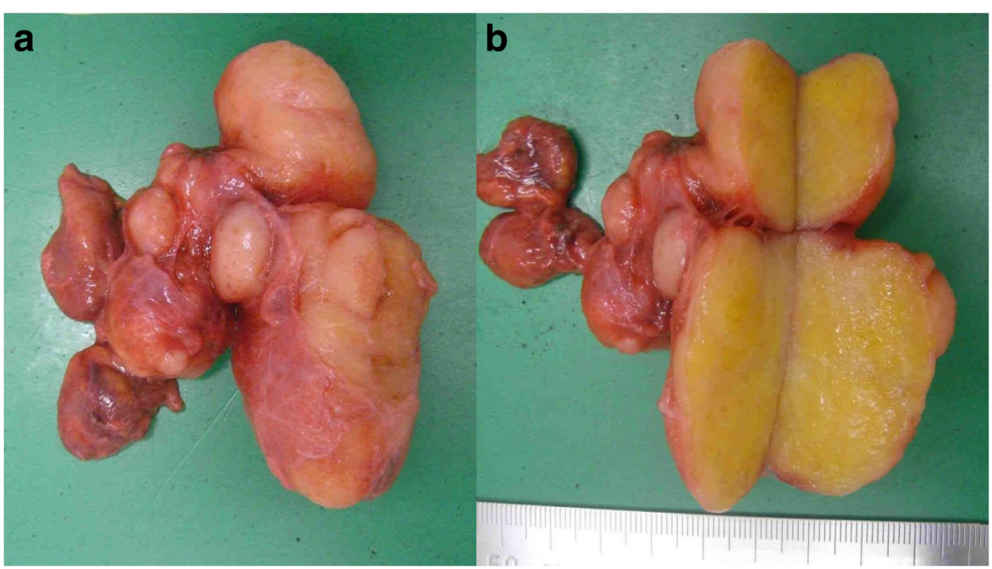

Fig. 6 Surgical specimen. a The specimen showed a well-demarcated, elastic hard, and lobulated appearance and was measured $80 \times 42 \mathrm{~mm}$. b The cut surface was almost uniformly pale yellow

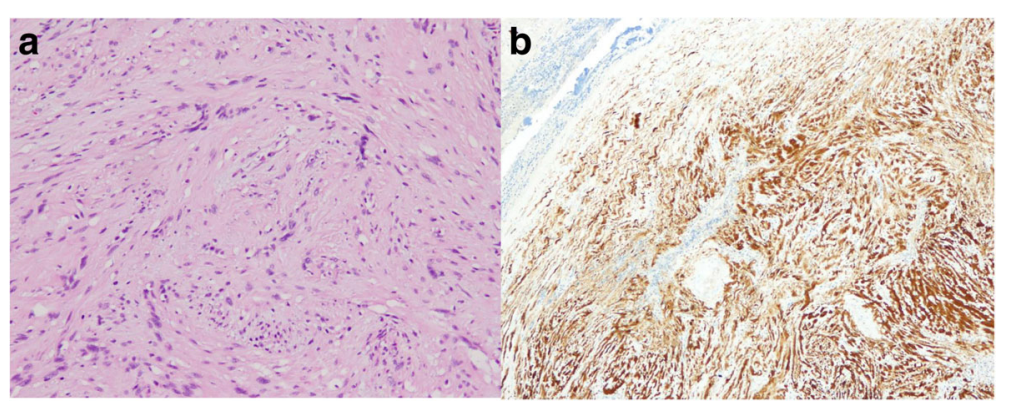

Fig. 7 Histopathological examination. a Hematoxylin and eosin staining revealed spindle-shaped cells configuring plexiform proliferation. b Immunohistochemical examination revealed S-100 protein positivity 


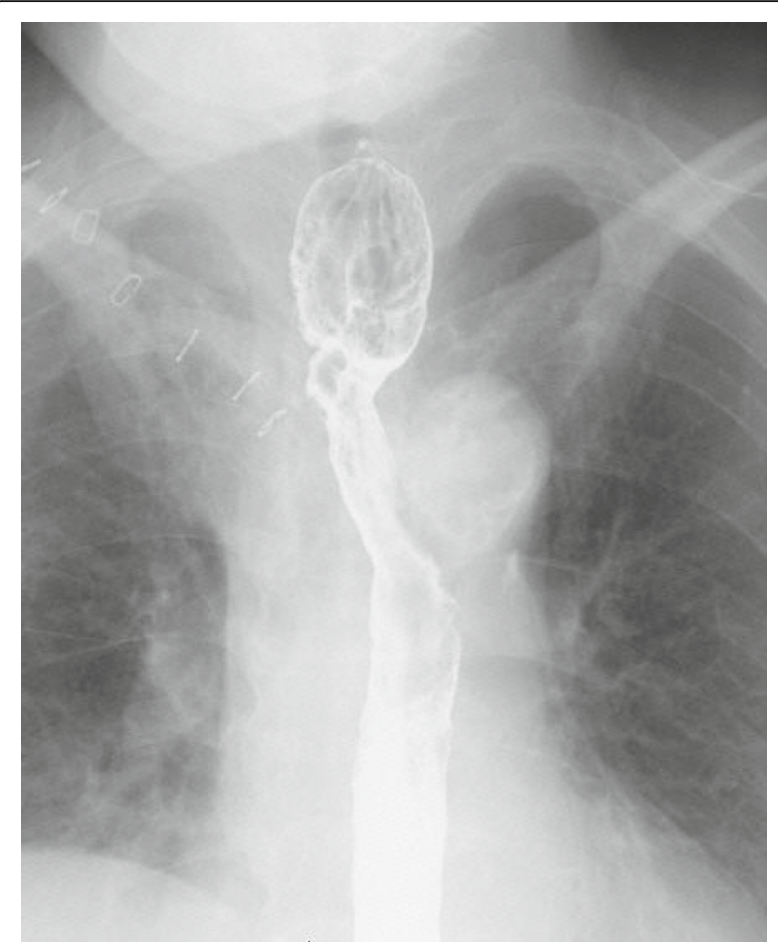

Fig. 8 Upper gastrointestinal series. Upper gastrointestinal series showed no leakage, slight stenosis in the upper thoracic esophagus, and dilatation in cervical esophagus

with endoscopy every year. No recurrences have seen for 5 years after surgery.

\section{Conclusions}

We experienced a case of lobulated esophageal schwannoma with FDG accumulation. We resected the tumor with concurrent approach from the thorax and cervix.

\section{Acknowledgements}

Not applicable

\section{Funding}

The authors have no conflicts of relevant financial interests.

\section{Availability of data and materials}

Not applicable.

\section{Authors' contributions}

YI wrote the paper. CT and NN studied the concept and patient care. MK, KK, and KY studied the concept of the study. All authors read and approved the final manuscript.

\section{Ethics approval and consent to participate}

Not applicable.

\section{Consent for publication}

Not applicable.

\section{Competing interests}

The authors declare that they have no competing interests.

\section{Publisher's Note}

Springer Nature remains neutral with regard to jurisdictional claims in published maps and institutional affiliations.

\section{Author details}

${ }^{1}$ Department of Surgery, Gifu Prefectural General Medical Center, 4-6-1

Noisshiki, Gifu, Japan. ${ }^{2}$ Department of Surgical Oncology, Gifu University

School of Medicine, 1-1 Yanagido, Gifu, Japan.

Received: 30 July 2017 Accepted: 5 February 2018

Published online: 13 February 2018

\section{References}

1. Choong CK, Meyers BF. Benign esophageal tumors: introduction, incidence, classification, and clinical features. Semin Thorac Cardiovasc Surg. 2003;15(1):3-8.

2. Matsuki A, Kosugi S, Kanda T, Komukai S, Ohashi M, Umezu H, et al. Schwannoma of the esophagus: a case exhibiting high 18Ffluorodeoxyglucose uptake in positron emission tomography imaging. Dis Esophagus. 2009;22(4):E6-e10. https://doi.org/10.1111/j.1442-2050. 2007.00712.x.

3. Naus PJ, Tio FO, Gross GW. Esophageal schwannoma: first report of successful management by endoscopic removal. Gastrointest Endosc. 2001; 54(4):520-2.

4. Kent M, d'Amato T, Nordman C, Schuchert M, Landreneau R, Alvelo-Rivera M, et al. Minimally invasive resection of benign esophageal tumors. J Thorac Cardiovasc Surg. 2007;134(1):176-81. https://doi.org/10.1016/j.jtcvs.2006.10.082.

5. Kitada M, Matsuda Y, Hayashi S, Ishibashi K, Oikawa K, Miyokawa N. Esophageal schwannoma: a case report. World J Surg Oncol. 2013;11:253. https://doi.org/10.1186/1477-7819-11-253.

6. Kassis ES, Bansal S, Perrino C, Walker JP, Hitchcock C, Ross P Jr, et al. Giant asymptomatic primary esophageal schwannoma. Ann Thorac Surg. 2012; 93(4):e81-3. https://doi.org/10.1016/j.athoracsur.2011.10.054.

7. Dutta R, Kumar A, Jindal T, Tanveer N. Concurrent benign schwannoma of oesophagus and posterior mediastinum. Interact Cardiovasc Thorac Surg. 2009:9(6):1032-4. https://doi.org/10.1510/icvts.2009.216440.

8. Winant AJ, Gollub MJ, Shia J, Antonescu C, Bains MS, Levine MS. Imaging and clinicopathologic features of esophageal gastrointestinal stromal tumors. AJR Am J Roentgenol. 2014;203(2):306-14. https://doi. org/10.2214/ajr.13.11841.

9. Shinagare AB, Zukotynski KA, Krajewski KM, Jagannathan JP, Butrynski J, Hornick $J$, et al. Esophageal gastrointestinal stromal tumor: report of 7 patients. Cancer Imaging. 2012;12:100-8. https://doi.org/10.1102/14707330.2012.0017.

10. Watanabe T, Miyazaki T, Saito H, Yoshida T, Kumakura Y, Honjyo H, et al. Resection of an esophageal schwannoma with thoracoscopic surgery: a case report. Surg Case Rep. 2016;2(1):127. https://doi.org/10. 1186/s40792-016-0256-0.

11. Liu D, Yang Y, Qi YU, Wu K, Zhao S. Schwannoma of the esophagus: a case report. Oncol Lett. 2015;10(5):3161-2. https://doi.org/10.3892/ol.2015.3659.

12. Jeon HW, Kim KS, Hyun KY, Park JK. Enucleation of giant esophageal schwannoma of the upper thoracic esophagus: reports of two cases. World J Surg Oncol. 2014;12:39. https://doi.org/10.1186/1477-7819-12-39.

13. Beaulieu S, Rubin B, Djang D, Conrad E, Turcotte E, Eary JF. Positron emission tomography of schwannomas: emphasizing its potential in preoperative planning. AJR Am J Roentgenol. 2004;182(4):971-4. https://doi. org/10.2214/ajr.182.4.1820971.

14. Kobayashi N, Kikuchi S, Shimao H, Hiki Y, Kakita A, Mitomi H, et al. Benign esophageal schwannoma: report of a case. Surg Today. 2000;30(6):526-9. https://doi.org/10.1007/s005950070120.

15. Mizuguchi S, Inoue K, Imagawa A, Kitano Y, Kameyama M, Ueda H, et al. Benign esophageal schwannoma compressing the trachea in pregnancy. Ann Thorac Surg. 2008:85(2):660-2. https://doi.org/10.1016/j. athoracsur.2007.07.088. 